\title{
Random Matrix Theory Filters in Portfolio Optimisation: A Stability and Risk Assessment
}

\author{
J. Daly, M. Crane, H. J. Ruskin \\ Dublin City University, Glasnevin, Dublin 9, Ireland
}

\begin{abstract}
Random matrix theory (RMT) filters, applied to covariance matrices of financial returns, have recently been shown to offer improvements to the optimisation of stock portfolios. This paper studies the effect of three RMT filters on realised portfolio risk, and on the stability of the filtered covariance matrix, using bootstrap analysis and out-of-sample testing.
\end{abstract}

We propose an extension to an existing RMT filter, (based on Krzanowski stability), which is observed to reduce risk and increase stability, compared to other RMT filters tested. We also study a scheme for filtering the covariance matrix directly, as opposed to the standard method of filtering correlation, where the latter is found to lower realised risk on average, by up to $6.7 \%$.

We consider both equally and exponentially weighted covariance matrices in our analysis, and observe that the overall best method out-of-sample was that of exponentially weighted covariance, with our Krzanowski stability-based filter applied to the correlation matrix. We also find that the optimal out-of-sample decay factors, for both filtered and unfiltered forecasts, were higher than those suggested by Riskmetrics [1], with those for the latter approaching a value of $\alpha=1$.

In conclusion, RMT filtering reduced realised risk on average, and in the majority of cases, when tested out-of-sample, but increased realised risk on a marked number of individual days, in some cases more than doubling it.

Key words: Random matrix theory; Portfolio optimisation; Econophysics PACS: 05.40.-a; 05.45.Tp; 89.65.Gh

Email address: jdaly@computing.dcu.ie, mcrane@computing.dcu.ie, hruskin@computing.dcu.ie (J. Daly, M. Crane, H. J. Ruskin). 


\section{Introduction}

Markowitz portfolio theory [2], an intrinsic part of modern financial analysis, relies on the covariance matrix of returns and this can be difficult to estimate. For example, for a time series of length $T$, a portfolio of $N$ assets requires $\left(N^{2}+\right.$ $N) / 2$ covariances to be estimated from $N T$ returns. This results in estimation noise, since the availability of historical information is limited. Moreover, it is commonly accepted that financial covariances are not fixed over time (e.g. $[1,3,4])$ and thus older historical data, even if available, can lead to cumulative noise effects.

Random matrix theory (RMT), first developed by authors such as Dyson and Mehta [5-8], to explain the energy levels of complex nuclei [9], has recently been applied to noise filtering in financial time series, particularly in large dimensional systems such as stock markets, by several authors including Plerou et al. [9-13] and Laloux et al. [14,15]. Both groups have analysed US stock markets and have found that the eigenvalues of the correlation matrix of returns are consistent with those calculated using random returns, with the exception of a few large eigenvalues. Moreover, their findings indicated that these large eigenvalues, which do not conform to random returns, had eigenvectors that were more stable over time. Of particular interest was the demonstration $[9,15]$, that filtering techniques, based on RMT, could be beneficial in portfolio optimisation, both reducing the realised risk of optimised portfolios, and improving the forecast of this realised risk.

More recently, Pafka et al. [16] extended RMT to provide Riskmetrics type [1] covariance forecasts. Riskmetrics, dating from the 1990's and considered a benchmark in Risk management [16], uses an exponential weighting to model the heteroskedasticity of financial returns. Pafka et al. [16] showed that RMTbased eigenvalue filters can improve the optimisation of minimum risk portfolios, generated using exponentially weighted forecasts. However, these authors found that the decay factors which produced the least risky portfolios were higher than the range suggested by Riskmetrics and further concluded that unfiltered Riskmetrics-recommended forecasts were unsuitable for their portfolio optimisation problem. A recent paper by Sharifi et al. [17], using equally weighted, high frequency returns for estimating covariances, proposed an alternative eigenvalue-filtering method, based on a principal components technique developed by Krzanowski [18] for measuring the stability of eigenvectors, in relation to small perturbations in the corresponding eigenvalues. Sharifi et al. [17] concluded that filtering correlation matrices according to the method outlined in Laloux et al. [15] had a negative effect on this stability.

Our objectives in this article are (i) to present a computationally efficient method of calculating the maximum eigenvalue of an exponentially weighted 
random matrix (ii) to study the behaviour of the stability-based filter [17] for daily data and for exponentially weighted covariance (iii) to explore the possibility of filtering the covariance matrix directly (as opposed to the standard method of filtering correlation) and (iv) to compare three available RMT filters using bootstrapping and out-of-sample testing. The paper is organised as follows. In Section 2, we review the theoretical background for the three RMT filters, Section 3 contains the in-sample analysis of the filters from a stability and risk reduction perspective, and in Section 4 we present results of the outof-sample test on effectiveness of the filters, in reducing risk. In Appendix A, we describe the filtering methods of Laloux et al. [15] and Plerou et al. [9].

\section{Background}

\subsection{Random Matrix Theory and Historical Covariance}

As described by Laloux et al. [14], Plerou et al. [9], Sharifi et al. [17] and others, in the context of correlation matrices of financial returns, if $\mathbf{R}$ is any matrix defined by

$$
\mathbf{R}=\frac{1}{T} \mathbf{A A}^{\prime}
$$

where $\mathbf{A}$ is an $N \times T$ matrix whose elements are i.i.d. ${ }^{1}$ random variables with zero mean, then it has been shown [19] that, in the limit $N \rightarrow \infty, T \rightarrow \infty$ such that $Q=T / N \geq 1$ is fixed, the distribution $P(\lambda)$ of the eigenvalues of $\mathbf{R}$ is self-averaging, and is given by

$$
P(\lambda)= \begin{cases}\frac{Q}{2 \pi \sigma^{2}} \frac{\sqrt{\left(\lambda_{+}-\lambda\right)\left(\lambda-\lambda_{-}\right)}}{\lambda} & \text { if } \lambda_{-} \leq \lambda \leq \lambda_{+} \\ 0 & \text { otherwise }\end{cases}
$$

where $\sigma^{2}$ is the variance of the elements of $\mathbf{A}$ and

$$
\lambda_{ \pm}=\sigma^{2}(1+1 / Q \pm 2 \sqrt{1 / Q}) .
$$

Financial correlation and covariance matrices can be expressed, in general, in the form given by Equation (1), so matrices for historical data can be compared

$\overline{1 \text { i.i.d. }} \equiv$ independent and identically distributed 
to those generated from random returns. Here we define the covariance matrix $\mathbf{V}=\left\{\sigma_{i j}\right\}_{i, j=1}^{N}$ of returns ${ }^{2}$ by

$$
\sigma_{i j}=\left\langle G_{i}(t) G_{j}(t)\right\rangle-\left\langle G_{i}(t)\right\rangle\left\langle G_{j}(t)\right\rangle
$$

where $\langle\cdot\rangle$ refers to the mean over time, and the correlation matrix $\mathbf{C}=$ $\left\{\rho_{i j}\right\}_{i, j=1}^{N}$ is given by

$$
\rho_{i j}=\sigma_{i j} / \sqrt{\sigma_{i i} \sigma_{j j}}
$$

where $\left\{G_{i}(t)\right\}_{t=1, \ldots, T}^{i=1, \ldots, N}$ are the returns

$$
G_{i}(t)=\ln \left(S_{i}(t) / S_{i}(t-1)\right)
$$

and where $S_{i}(t)$ is the spot price of asset $i$ at time $t$.

\subsection{Random Matrix Theory and Exponentially Weighted Covariance}

In extending RMT filtering to exponentially weighted matrices, Pafka et al. [16] have analysed matrices of the form $\mathbf{M}=\left\{m_{i j}\right\}_{i, j=1}^{N}$ with

$$
m_{i j}=\sum_{k=0}^{\infty}(1-\alpha) \alpha^{k} x_{i k} x_{j k}
$$

and where $\left\{x_{i k}\right\}_{k=0, \ldots, \infty}^{i=1, \ldots, N}$ are assumed to be N.I.D. $\left(0, \sigma^{2}\right) .{ }^{3}$ They have shown that, in the special case $N \rightarrow \infty, \alpha \rightarrow 1$ with $Q \equiv 1 /(N(1-\alpha))$ fixed, the density, $\rho(\lambda)$, of the eigenvalues of $\mathbf{M}$ is given by $\rho(\lambda)=Q v / \pi$ where $v$ is the root of

$$
F(v)=\frac{\lambda}{\sigma^{2}}-\frac{v \lambda}{\tan (v \lambda)}+\ln \left(v \sigma^{2}\right)-\ln (\sin (v \lambda))-\frac{1}{Q}
$$

$F(v)$ is well defined on the open interval $(0, \pi / \lambda)$. If a root does not exist on this interval for a given value of $\lambda$ we define $\rho(\lambda)=0$ for that $\lambda$. The family of matrices, defined by Equation (7), includes the Riskmetrics [1] covariance

$\overline{2}$ throughout this paper the following notation is used: $\left\{x_{i}\right\}_{i=1}^{N} \equiv\left\{x_{i}: i=\right.$ $1, \ldots, N\},\left\{x_{i j}\right\}_{i, j=1}^{N} \equiv\left\{x_{i j}: i=1, \ldots, N ; j=1, \ldots, N\right\},\left\{x_{i t}\right\}_{t=1, \ldots, T}^{i=1, \ldots, N} \equiv\left\{x_{i t}:\right.$ $i=1, \ldots, N ; t=1, \ldots, T\}$ etc.

3 N.I.D. $\left(\mu, \sigma^{2}\right) \equiv$ Normally and identically distributed (with mean $\mu$ and variance $\left.\sigma^{2}\right)$ 
and correlation matrices. Following this, we define the exponentially weighted covariance matrix $\mathbf{V}^{*}=\left\{\sigma_{i j}^{*}\right\}_{i, j=1}^{N}$ by

$$
\sigma_{i j}^{*}=\frac{1-\alpha}{1-\alpha^{T}} \sum_{t=0}^{T-1} \alpha^{t}\left(G_{i}(T-t)-\left\langle G_{i}\right\rangle\right)\left(G_{j}(T-t)-\left\langle G_{j}\right\rangle\right)
$$

and define the corresponding, exponentially weighted, correlation matrix $\mathbf{C}^{*}=$ $\left\{\rho_{i j}^{*}\right\}_{i, j=1}^{N}$ by

$$
\rho_{i j}^{*}=\sigma_{i j}^{*} / \sqrt{\sigma_{i i}^{*} \sigma_{j j}^{*}}
$$

Here, $\alpha$ is commonly called the decay factor.

\subsection{Maximum Eigenvalue of an Exponentially Weighted Random Matrix}

The maximum eigenvalue of an exponentially weighted random matrix can be found using Equation (8), but a more efficient method can be derived as follows. On the interval $v \in(0, \pi / \lambda)$, the following limits hold

$$
\begin{aligned}
\lim _{v \rightarrow 0} F(v) & =\frac{\lambda}{\sigma^{2}}-\ln \left(\frac{\lambda}{\sigma^{2}}\right)-\frac{1}{Q}-1 \\
\lim _{v \rightarrow(\pi / \lambda)} F(v) & =\infty
\end{aligned}
$$

Moreover, $F(v)$ is increasing on the interval $v \in(0, \pi / \lambda)$, since for $x=v \lambda$

$$
\begin{aligned}
F^{\prime}(v) & =\frac{1}{v}-\frac{x}{v}\left(\frac{2 \tan (x)-x \sec ^{2}(x)}{\tan ^{2}(x)}\right) \\
& =\frac{1}{v}\left(\frac{\sin ^{2}(x)-2 x \sin (x) \cos (x)+x^{2}}{\sin ^{2}(x)}\right)
\end{aligned}
$$

and also $h(x)=\sin ^{2}(x)-2 x \sin (x) \cos (x)+x^{2}>0$ on $x \in(0, \pi)$, which is true because $h(0)=0$ and $h^{\prime}(x)=4 x \sin ^{2}(x)>0$.

Therefore, a root of $F(v)$ exists on $v \in(0, \pi / \lambda)$ for a given $Q$ and $\lambda$ when its lower limit is negative on the interval, i.e. when

$$
\frac{\lambda}{\sigma^{2}}-\ln \left(\frac{\lambda}{\sigma^{2}}\right)<1+\frac{1}{Q}
$$


Now, as seen from Figure $1, \lambda / \sigma^{2}-\ln \left(\lambda / \sigma^{2}\right) \geq 1$, with a minimum at 1 when $\lambda=\sigma^{2}$, and it crosses $1+1 / Q>1$ just once above $\lambda=\sigma^{2}$ and once below it. Outside of the open interval, bracketed by these crossovers, we have

$$
\frac{\lambda}{\sigma^{2}}-\ln \left(\frac{\lambda}{\sigma^{2}}\right) \geq 1+\frac{1}{Q}
$$

and thus $F(v)$ cannot have a root on $v \in(0, \pi / \lambda)$. It follows that these crossovers are the minimum and maximum possible eigenvalues for the exponentially weighted random matrix. Thus, the theoretical maximum eigenvalue

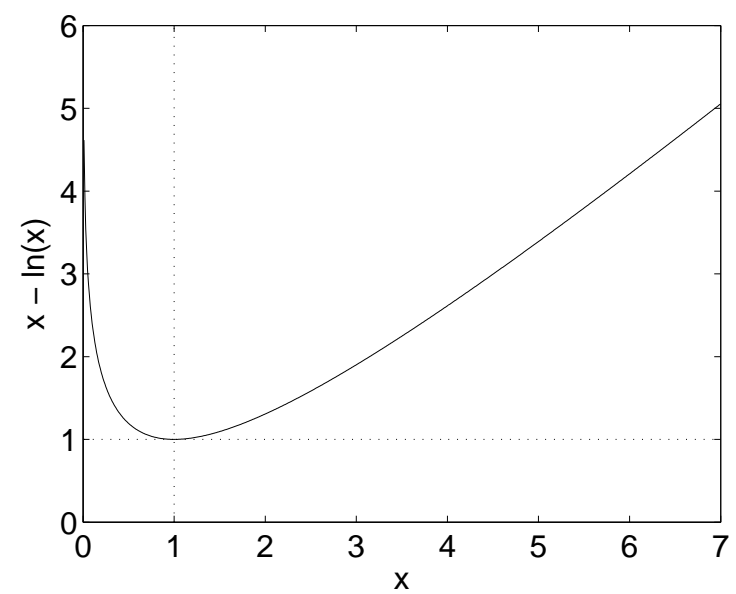

Fig. 1. $x-\ln (x)$

is the solution of

$$
\frac{\lambda}{\sigma^{2}}-\ln \left(\frac{\lambda}{\sigma^{2}}\right)=1+\frac{1}{Q}, \quad \lambda>\sigma^{2}
$$

We note also that Potters et al. [20] have demonstrated an alternative derivation, with $\sigma=1$, using "Blue" functions.

\subsection{Krzanowski Stability}

One of the filtering methods discussed, Sharifi et al. [17], and considered also here, is based on the stability, as described by Krzanowski [18], of the filtered matrix. Krzanowski [18] measured eigenvector stability, specifically the effect on each eigenvector of a perturbation in the corresponding eigenvalue. This is in contrast to stability over time, as analysed by many other authors, e.g. [9, 15]. Krzanowski [18] considered the angle, $\theta_{i}$, between an eigenvector $v_{i}$ and $v_{i}^{p}$, where $v_{i}^{p}$ is the maximum perturbation that can be applied to $v_{i}$ while ensuring 
that the eigenvalue, $\lambda_{i}^{p}$, corresponding to $v_{i}^{p}$ is within $\epsilon$ of the eigenvalue, $\lambda_{i}$, corresponding to $v_{i}$. He showed that $\theta_{i}$ is given by:

$$
\cos \theta_{i}= \begin{cases}\left(1+\frac{\epsilon}{\lambda_{i}-\lambda_{i-1}}\right)^{-\frac{1}{2}} & \text { for } \lambda_{i}^{p}<\lambda_{i} \\ \left(1+\frac{\epsilon}{\lambda_{i+1}-\lambda_{i}}\right)^{-\frac{1}{2}} & \text { for } \lambda_{i}<\lambda_{i}^{p}\end{cases}
$$

where $\lambda_{1} \leq \lambda_{2} \leq \ldots \leq \lambda_{L}$ are the eigenvalues, and suggested using $\epsilon=k \lambda_{i}$ with $k=0.1,0.05$ or 0.01 . We have chosen $k=0.1$, which was the most consistent with typical eigenvalue changes between different subperiods of our data. When measuring mean stability, of the filtered and unfiltered covariance matrices, the arithmetic mean of the cases $\lambda_{i}^{p}<\lambda_{i}$ and $\lambda_{i}<\lambda_{i}^{p}$ was calculated.

\subsection{Filtering Methods}

All three filtering methods compared here are based on replacing the "noisy" eigenvalues of the covariance or correlation matrix, while maintaining its trace. The noisy eigenvalues are taken to be those that are less than or equal to the maximum possible eigenvalue of the corresponding random matrix. The theoretical limiting cases (described, Sections 2.1 and 2.2) are commonly used to estimate the maximum eigenvalues of the random matrices. However, these can also be estimated by calculating them directly from Monte Carlo simulated random returns (for example if the number of assets is small). In this work, we have used "first order filtering", i.e. exactly the maximum eigenvalue predicted by RMT. Improvement to these filters can potentially be made, by adjusting these limits to take account of the largest measured eigenvalues (generally known not to be random). For further details see, e.g., Laloux et al [15].

The filtering methods of Laloux et al. [15] (referred to hereafter as LCPB), and of Plerou et al. [9] (referred to hereafter as PG+), are detailed in Appendix A. The third filtering method is defined as follows. To maximise the Krzanowski stability of the filtered matrix, while also maintaining its trace, the method of Sharifi et al. [17] replaces the noisy eigenvalues with ones that are equally and maximally spaced, are positive, and have sum equal to the sum of those replaced. To achieve maximal spacing, it was assumed that the smallest replacement eigenvalue should be very close to zero. In this paper, this method is adapted by making the smallest replacement eigenvalue a parameter of the filter, so that changes in stability and optimisation results, achieved for various values of this parameter, can be measured. We call the adapted version the KR method.

The KR method is identical to the LCPB method except in the choice of 
eigenvalues to replace the noisy eigenvalues. If $\Lambda_{\text {noisy }}=\left\{y_{i}\right\}_{i=1}^{n}$ are the original noisy eigenvalues then for the KR method the replacement eigenvalues $\Lambda_{\text {new }}=\left\{x_{i}\right\}_{i=1}^{n}$ are given by $x_{i}=x_{1}+(i-1) k$ for some constant $k$, defined by the choice of minimum replacement eigenvalue $x_{1}$ and the constraint that the sum of the replacement eigenvalues must equal the sum of the eigenvalues being replaced. In addition, the replacement eigenvalues must all be strictly positive. It follows that $k=2\left(a-x_{1}\right) /(n-1)$ where $a$ is the mean of the eigenvalues being replaced. The cases $k>0$ and $k<0$ can be shown to be equivalent, so we can assume without loss of generality that $x_{1} \leq a$. Moreover, the case $k=0$ just collapses to the LCPB method (as defined in Appendix A), since $k=0 \Leftrightarrow x_{1}=a$.

\subsection{Data}

The data, used to test filter performance, were daily closing prices for the S\&P 500 index stocks, with the index composition taken as of 1st February $2006^{4}$. The dataset runs from 1st June 1995 to 1st February 2006, and any series not covering the entire period were discarded, leaving a total of 432 stocks.

\section{In Sample Evaluations}

\subsection{Evaluation Methods}

For the in-sample analysis, and following [16], bootstrapped samples were taken, together with the mean across these samples. For a given value of $N$ (the number of assets) and $F$ (the forecast horizon in working days), we randomly selected $N$ assets from the data set, and a random test date. Everything up to and including the test date was taken as historical information and everything afterwards as realised, future information. For the same $N$ and $F$, we repeated this random selection 1000 times, with replacement, and calculated the mean, across all bootstrapped samples, of (i) the realised risk of the forecast minimum risk portfolio [16] (calculated using our forecast covariance) and (ii) the mean Krzanowski stability [18] across all eigenvectors of the forecast covariance matrix. We analysed the cases $N=100,250$ and 432, and $F=20$ and 50. The results for $F=50$ were very similar to those for $F=20$ and thus we concentrated subsequently on the $F=20$ case.

A covariance forecast in this context consisted of a raw forecast, which was

$\overline{4}$ from www.standardandpoors.com 
either exponentially or equally weighted, and could be unfiltered, or filtered by one of the LCPB, PG+ or KR methods applied to either the covariance or correlation matrix. In much of the literature (e.g. $[9,15])$ the correlation matrix is filtered, it being closer to the RMT assumption of i.i.d. returns than the covariance matrix. Here, we also wish to assess the impact of filtering covariance directly, since it retains more information about the individual assets.

On each test date, we calculated the forecast minimum risk portfolio, optimised as follows [16]. Choose a portfolio weighting $\left\{w_{i}\right\}_{i=1}^{N}$ that minimises

$$
\sum_{i, j=1}^{N} w_{i} w_{j} \hat{\sigma}_{i j}
$$

while satisfying the budget constraint

$$
\sum_{i=1}^{N} w_{i}=1
$$

Here, $\hat{\mathbf{V}}=\left\{\hat{\sigma}_{i j}\right\}_{i, j=1}^{N}$ is one of the 14 forecast covariance matrices. The solution, $\left\{\hat{w}_{i}\right\}_{i=1}^{N}$, of this problem is:

$$
\hat{w}_{i}=\frac{\sum_{j=1}^{N} \hat{\sigma}_{i j}^{-1}}{\sum_{j, k=1}^{N} \hat{\sigma}_{j k}^{-1}} \forall i
$$

where $\hat{\mathbf{V}}^{-1}=\left\{\hat{\sigma}_{i j}^{-1}\right\}_{i, j=1}^{N}$ is the matrix inverse of $\hat{\mathbf{V}}$. The realised risk of the optimal portfolio is defined by

$$
\sqrt{\sum_{i, j=1}^{N} \hat{w}_{i} \hat{w}_{j} \tilde{\sigma}_{i j}}
$$

Here, $\tilde{\mathbf{V}}=\left\{\tilde{\sigma}_{i j}\right\}_{i, j=1}^{N}$ is the realised covariance matrix, and is just the (equally weighted) covariance matrix of the realised future returns over the investment period. The forecast risk is calculated analogously, using the forecast covariance matrix, $\hat{\mathbf{V}}$.

By comparing the covariance forecasts in this way, we measure their effect on realised risk without using forecast returns, which would introduce unwanted noise into the results. Further, we have not used any knowledge of future returns in our tests, since we wished to evaluate both forecasting methods (equal vs. exponential weighting) as well as filtering methods. This is in contrast to 
some previous studies, that have isolated the effect of the filtering method on the correlation matrix, by using future knowledge of realised returns to estimate the variance of each individual asset.

\subsection{Measuring Noise}

Figure 2 shows, for equally and exponentially weighted forecasts, and for 100 and 432 assets, the percentage of measured eigenvalues, for both covariance and correlation forecasts, that were larger than the corresponding maximum eigenvalue predicted by RMT. It can be seen that, in general, compared to the correlation matrix, the covariance matrix contained more "non-random" eigenvalues. In the case of exponentially weighted matrices with 432 assets however, the effect was less pronounced. For a wide range of decay factor values, expo-

(a)

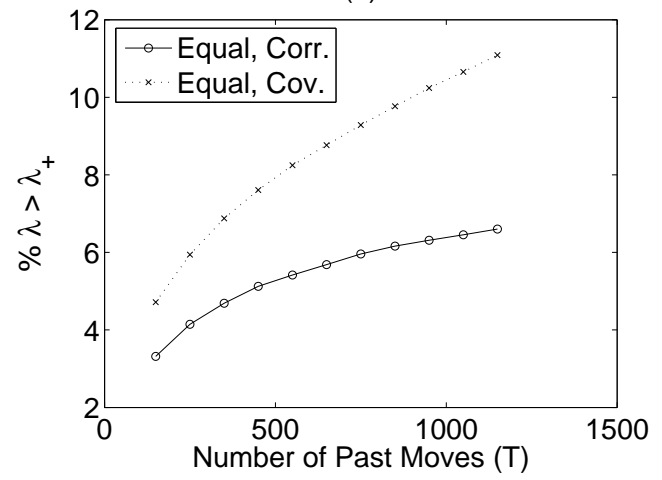

(c)

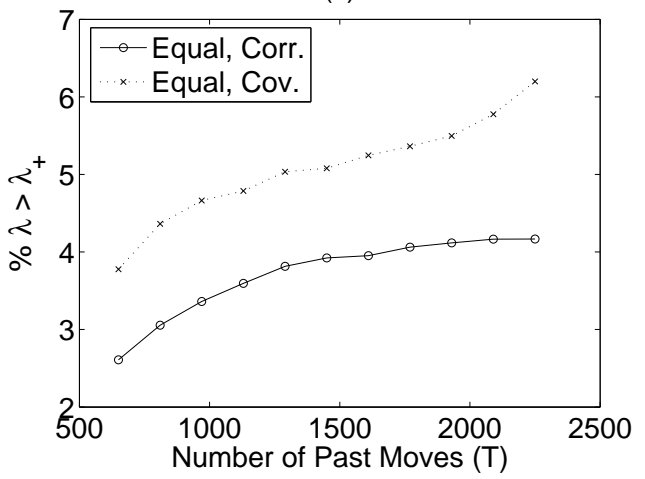

(b)

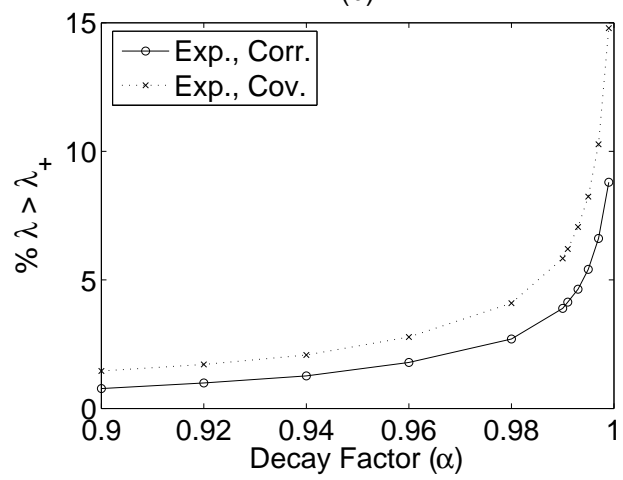

(d)

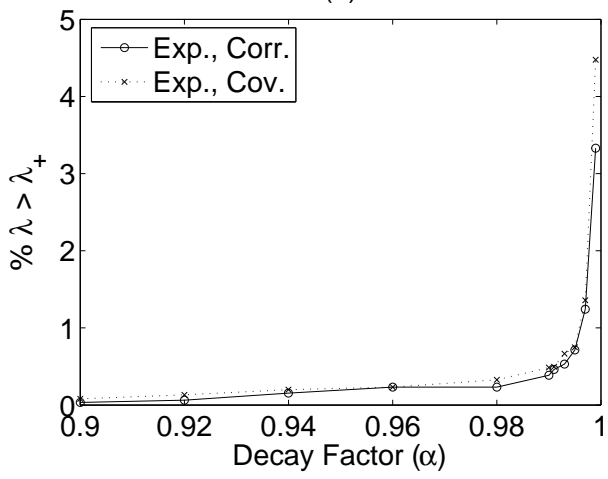

Fig. 2. Percentage of eigenvalues that are larger than the maximum eigenvalue predicted by RMT, for equally weighted correlation and covariance with (a) 100 assets and (c) 432 assets, and for exponential weights with (b) 100 assets and (d) 432 assets.

nentially weighted matrices had very few non-noisy eigenvalues when all 432 assets were used. The range for $\alpha$ included values suggested by Riskmetrics [1] (0.94 to 0.97 ). The high level of measured noise reflects that lowering the value of the decay factor is equivalent to using less (equally weighted) data. Despite 
this, the filtered exponentially weighted forecasts produced some of the lowest mean realised risks.

\subsection{Stability}

Focusing on stability, Figure 3 displays, for selected filters, and averaged over all bootstrap samples, the mean stability across all eigenvectors of the filtered covariance matrix, (for the case of 100 assets, and using equally weighted forecasts). These results are representative of the stability results for other sizes of asset group, and for exponential weights. The covariance matrices produced by KR filtering are seen to have better stability than the LCPB and PG+ filtered ones, particularly for direct filtering of the covariance matrix. The KR methods considered, (and their defining minimum replacement eigenvalues), were: $\operatorname{KR2}\left(\frac{1}{2} \bar{\Lambda}_{\text {noisy }}\right), \operatorname{KR} 4\left(\frac{1}{4} \bar{\Lambda}_{\text {noisy }}\right), \operatorname{KR} 8\left(\frac{1}{8} \bar{\Lambda}_{\text {noisy }}\right), \operatorname{KR} 16\left(\frac{1}{16} \bar{\Lambda}_{\text {noisy }}\right), \operatorname{KR} 64$ $\left(\frac{1}{64} \bar{\Lambda}_{\text {noisy }}\right), \operatorname{KR} 100\left(\frac{1}{100} \bar{\Lambda}_{\text {noisy }}\right)$ and $\operatorname{KR} 1000\left(\frac{1}{1000} \bar{\Lambda}_{\text {noisy }}\right)$, where $\bar{\Lambda}_{\text {noisy }}$ is the mean of the noisy eigenvalues. It can also be seen that stability improved as the minimum replacement eigenvalue for the KR filter approached zero. However, the closer the minimum eigenvalue approached to the mean noisy eigenvalue, the more stability decreased (though it remained above that of the $\mathrm{LCPB}$ and PG+ filters). These results are consistent with the definition of the KR filter, which is designed to give improved stability.
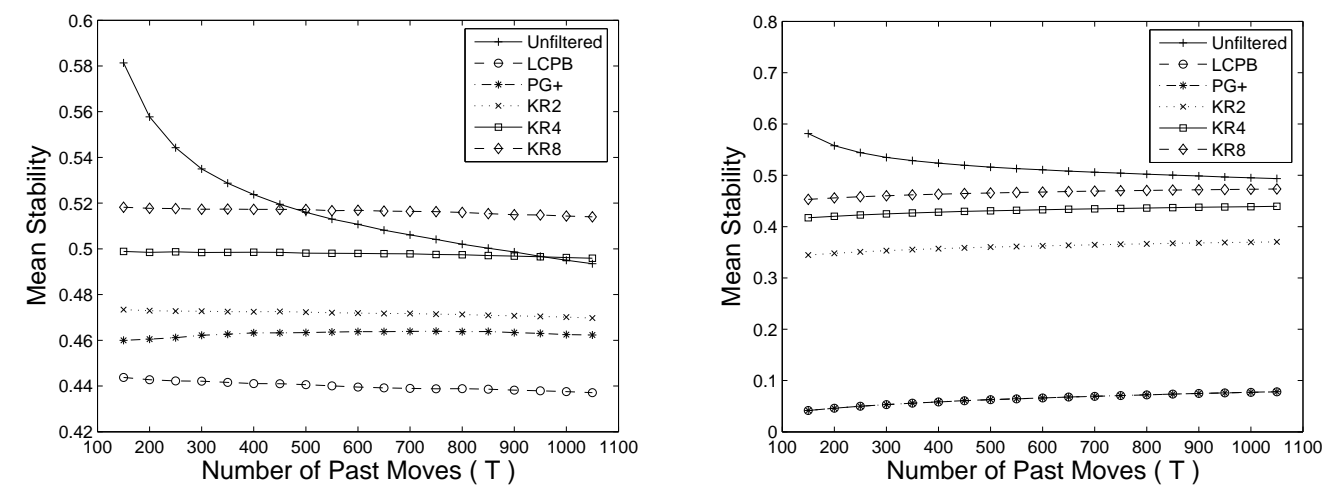

Fig. 3. In-sample mean stability for the equally weighted covariance forecast with 100 assets, filtering correlation (left) and covariance (right)

\subsection{In-Sample Risk Reduction}

We found that the KR method as described in Sharifi et al. [17] (i.e. with a minimum replacement eigenvalue of $10^{-8}$ ) was not competitive when compared to other methods for reducing risk, including comparison with the unfiltered series. Figure 4 shows a sample comparison of this method, (which we call 
method KR0), with the LCPB and unfiltered methods and it is clear that the KR0 method increases mean realised risk. We found a marked risk reduction

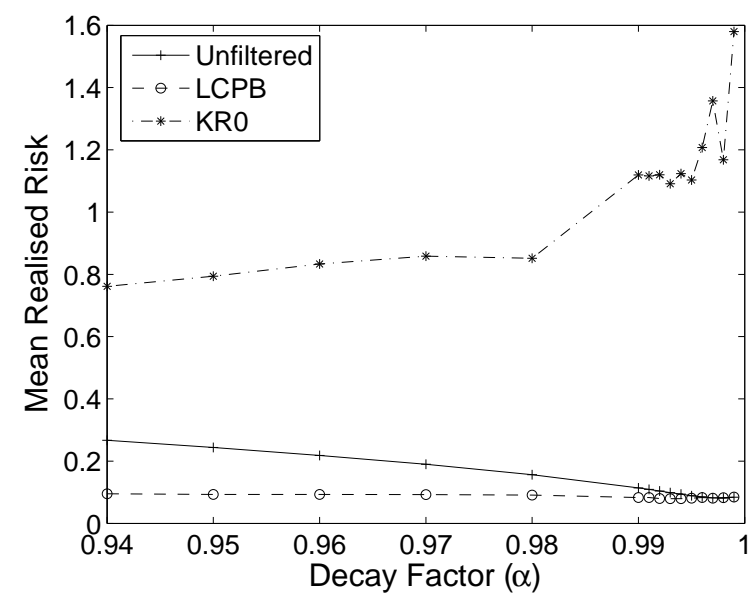

Fig. 4. In-sample mean realised risk for unfiltered, LCPB and KR0 filtered exponentially weighted forecasts, with 432 assets, (filtering covariance).

was achieved by varying the minimum replacement eigenvalue. The in-sample results showed, in general, the potential of RMT filters to reduce realised risk, and we noted, in particular, that the KR2, KR4 and KR8 methods were amongst the best performing of all filters for this, and were also reasonably consistent with each other. In general, optimisation performance of the KR filters disimproved as the minimum replacement eigenvalue approached zero.

In many cases, two local minima were produced for the choice of optimal decay factor for the exponential weights ${ }^{5}$. One of these coincided with the suggestion of Riskmetrics [1], i.e. 0.97 for monthly forecasts. The other was much closer to 1 . Figure 5 shows an extract of the in-sample results, namely the best, or two best, filtering methods, from the point of view of mean realised risk, in each of the following cases: equally weighted filtered correlation, equally weighted filtered covariance, exponentially weighted filtered correlation and exponentially weighted filtered covariance. Results for 100 assets (left) and 432 assets (right) are shown vs. decay factors $(\alpha)$ in the case of exponential weighting and vs. number of past moves $(T)$ in the case of equal weighting. The legends can be interpreted as "forecast weighting (equal or exponential), matrix filtered (correlation or covariance), filtering method". For example, "Equal, Corr, KR2" refers to the mean realised risk over all bootstrapped samples for equally weighted forecasts, filtered using method KR2 on the correlation matrix.

$\overline{5}$ optimal for reducing in-sample realised risk 

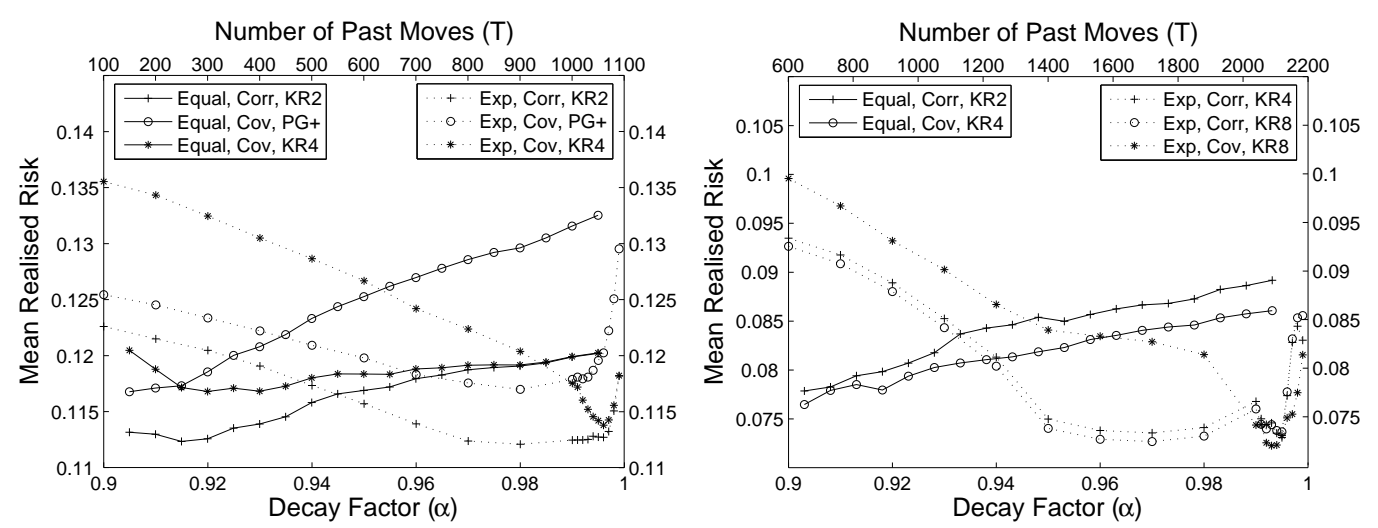

Fig. 5. In-sample mean realised risk for selected best forecasting methods, for 100 assets (left) and 432 assets (right)

\section{Out-of-Sample Testing}

For comparing the models out-of-sample we used forward validation. This method considers every available test date and for each one uses data prior to the test date to optimise any model parameters. This allows the comparison of filtering methods with different numbers of parameters and also gives some insight into the stability of the models over time. The value of the weighting parameter ( $\alpha$ or $T$ ) and the choice of KR model were determined outof-sample. Possible KR models were all KR models mentioned above (KR2, KR4, KR8, KR16, KR64, KR100, KR1000 and KR0) as well as the the LCPB model for completeness. The forward validation was performed over a period of 1658 days, 129 of which were used as the initial training period. Subsequent retraining was done daily. We used the realised risk of the forecast minimum risk portfolio as our metric and all 432 assets were used to eliminate the need to arbitrarily choose assets each day. Table 1 shows a summary of the performance of the covariance forecasting and filtering combinations. The figures shown are mean realised risk as a percentage of the result for unfiltered equally weighted covariance. The overall best performing combination in this test was exponential weighting with a KR filter applied to the correlation matrix (84\%). RMT filtering is seen on average to reduce realised risk in all cases where it is used.

Table 2 shows a breakdown of the mean realised risk of the various weightings and filters on an "annual" basis over 6 years. In this case a year is taken to have 255 trading days, with the final year having 254 . Here there are a few instances where filtering increases the mean risk in a year. However, the majority of the time filtering reduces risk. The overall best method was found to produce the lowest risk in three of the six years, and was competitive in the other three years. The stability-based KR filter is the only filter to reduce mean realised risk in all cases in each year. 
Table 1

Mean out-of-sample realised risk as a percentage of that for unfiltered equally weighted covariance. The weights (equal or exponential) are as described in Sections 2.1 and 2.2 and values are set using daily forward validation. Filtering is seen to reduce mean realised risk in all cases, and to as low as $84 \%$ of our benchmark risk, when applying KR filtering to the correlation matrix for exponentially weighted forecasts.

\begin{tabular}{ccccc}
\hline Model & Unfiltered & LCPB & PG+ & KR \\
\hline Equal Weights/Correlation Filtered & 100 & 87.3 & 87.6 & 85.1 \\
Exponential Weights/Correlation Filtered & 93.4 & 87.1 & 88.1 & 84 \\
Equal Weights/Covariance Filtered & 100 & 93.6 & 89.2 & 88.4 \\
Exponential Weights/Covariance Filtered & 93.4 & 92 & 87.1 & 86.7 \\
\hline
\end{tabular}

Table 2

Mean out-of-sample realised risk per year for 6 years as a percentage of equally weighted unfiltered result each year. In a few cases, RMT filtering is seen to increase mean realised risk over the course of a year. The KR filter is the only one to reduce realised risk in all cases each year.

\begin{tabular}{ccccccccc}
\hline Weights & Filtered & Filter & 1 & 2 & 3 & 4 & 5 & 6 \\
\hline Equal & No & - & 100 & 100 & 100 & 100 & 100 & 100 \\
Equal & Corr & LCPB & 86.3 & 86.5 & 89.8 & 85.3 & 94.6 & 81.3 \\
Equal & Corr & PG+ & 87.1 & 84.7 & 89.8 & 85.3 & 95.7 & 83.5 \\
Equal & Corr & KR & 84.2 & 84.7 & 87.4 & 83.2 & 91.4 & 80.2 \\
\hline Exp & No & - & 96.4 & 96.4 & 92.9 & 87.4 & 95.7 & 90.1 \\
Exp & Corr & LCPB & 81.3 & 82.9 & 89.8 & 85.3 & 94.6 & 91.2 \\
Exp & Corr & PG+ & 84.9 & 85.6 & 89 & 86.3 & 91.4 & 93.4 \\
Exp & Corr & KR & 80.6 & 81.1 & 89.8 & 80 & 88.2 & 85.7 \\
\hline Equal & No & - & 100 & 100 & 100 & 100 & 100 & 100 \\
Equal & Cov & LCPB & 95.7 & 100 & 96.1 & 87.4 & 91.4 & 89 \\
Equal & Cov & PG+ & 90.6 & 85.6 & 86.6 & 87.4 & 100 & 87.9 \\
Equal & Cov & KR & 89.2 & 91.9 & 92.1 & 83.2 & 87.1 & 84.6 \\
\hline Exp & No & - & 96.4 & 96.4 & 92.9 & 87.4 & 95.7 & 90.1 \\
Exp & Cov & LCPB & 92.1 & 95.5 & 99.2 & 85.3 & 84.9 & 93.4 \\
Exp & Cov & PG+ & 86.3 & 87.4 & 91.3 & 84.2 & 83.9 & 90.1 \\
Exp & Cov & KR & 89.2 & 89.2 & 90.6 & 81.1 & 83.9 & 83.5 \\
\hline
\end{tabular}


Table 3

Percentage of the time each method had the lowest mean out-of-sample realised risk on an annual, monthly and daily basis. Unfiltered forecasts are seen to have the lowest for only $6 \%$ of the days and for $5.5 \%$ of the months. The most consistent method was the stability-based filter, applied to the correlation matrix of the exponentially weighted forecasts.

\begin{tabular}{cccccc}
\hline Weights & Filtered & Filter & Yearly & Monthly & Daily \\
\hline Equal & No & - & 0 & 0 & 0.8 \\
Equal & Corr & LCPB & 0 & 4.1 & 7.1 \\
Equal & Corr & PG+ & 0 & 9.6 & 6.9 \\
Equal & Corr & KR & 16.7 & 8.2 & 7.7 \\
\hline Exp & No & - & 0 & 5.5 & 5.2 \\
Exp & Corr & LCPB & 0 & 6.2 & 4.9 \\
Exp & Corr & PG+ & 0 & 4.1 & 6.1 \\
Exp & Corr & KR & 50 & 25.3 & 19 \\
\hline Equal & No & - & 0 & 0 & 0.8 \\
Equal & Cov & LCPB & 0 & 4.1 & 3.3 \\
Equal & Cov & PG+ & 16.7 & 8.2 & 7.5 \\
Equal & Cov & KR & 0 & 2.7 & 4.6 \\
\hline Exp & No & - & 0 & 5.5 & 5.2 \\
Exp & Cov & LCPB & 0 & 6.8 & 6.9 \\
Exp & Cov & PG+ & 16.7 & 8.2 & 8.8 \\
Exp & Cov & KR & 0 & 6.8 & 11.2 \\
\hline
\end{tabular}

Table 3 shows the percentage of times that best performance was achieved by each method, on an annual, monthly and daily basis. One month is assumed equal to exactly 21 trading days for this purpose. The daily results show that an unfiltered forecast was best for only $6 \%$ of days, and on the majority of these days the best unfiltered forecast was exponentially weighted. The fact that unfiltered forecasting was found to be best for $5.5 \%$ of the months reflects some clustering of these daily effects. Overall, the best method was consistently so on a monthly $(25.3 \%)$ and daily $(19 \%)$ basis.

Table 4 shows the frequency of daily filtering effects. "Low" and "High" specify a range for the realised risk, expressed as a percentage of the relevant unfiltered realised risk. Methods L, P and $\mathrm{K}$ refer to the LCPB, PG+ and KR filters respectively. $\mathrm{L} / \mathrm{C}$ and $\mathrm{L} / \mathrm{V}$ refer to the $\mathrm{LCPB}$ filter applied to the correlation matrix and covariance matrix respectively and the $\mathrm{L}$ column is the sum of 
Table 4

Daily frequency of percentage effect, on realised risk, of applying RMT filters. "Low" and "High" specify a range for the realised risk, expressed as a percentage of the relevant unfiltered realised risk. Methods L, P and K refer to the LCPB, PG+ and $\mathrm{KR}$ filters. $\mathrm{L} / \mathrm{C}$ and $\mathrm{L} / \mathrm{V}$ refer to filtering the correlation and covariance matrices respectively. Filtering is seen to reduce realised risk $72.3 \%$ of the time overall, while stability-based filtering reduced it the most often, $80.5 \%$ of the time. It can also be seen that, despite reducing mean realised risk overall, all filters have the potential to markedly increase realised risk on any individual day.

\begin{tabular}{ccccccccccc}
\hline Low & High & L/C & P/C & K/C & L/V & P/V & K/V & L & $\mathrm{P}$ & $\mathrm{K}$ \\
\hline 40 & 60 & 38 & 45 & 25 & 10 & 42 & 5 & 48 & 87 & 30 \\
60 & 80 & 723 & 649 & 816 & 449 & 604 & 513 & 1172 & 1253 & 1329 \\
80 & 100 & 1431 & 1464 & 1626 & 1359 & 1523 & 1941 & 2790 & 2987 & 3567 \\
100 & 120 & 668 & 706 & 495 & 909 & 720 & 575 & 1577 & 1426 & 1070 \\
120 & 140 & 158 & 153 & 80 & 278 & 148 & 22 & 436 & 301 & 102 \\
140 & 160 & 30 & 32 & 11 & 36 & 15 & 2 & 66 & 47 & 13 \\
160 & 180 & 7 & 8 & 4 & 7 & 4 & 0 & 14 & 12 & 4 \\
180 & 200 & 3 & 1 & 1 & 8 & 2 & 0 & 11 & 3 & 1 \\
200 & 220 & 0 & 0 & 0 & 1 & 0 & 0 & 1 & 0 & 0 \\
220 & 240 & 0 & 0 & 0 & 1 & 0 & 0 & 1 & 0 & 0 \\
\hline
\end{tabular}

these. The effects on equally weighted and exponentially weighted matrices are combined to calculate $\mathrm{L} / \mathrm{C}, \mathrm{L} / \mathrm{V}$ etc. For example, the LCPB method reduced realised risk to $60 \%-80 \%$ of the unfiltered realised risk for 1172 of the 6116 daily tests. When only correlation filtering is accounted for LCPB filtering reduces to $60 \%-80 \%$ for 723 out of 3058 tests, 1529 equally weighted and 1529 exponentially weighted. From this we can see that, taking the mean across all filters, RMT filtering reduced realised risk on $72.3 \%$ of the days. This breaks down as $74.3 \%$ when correlation was filtered and $70.3 \%$ when covariance was filtered. The KR method was the most consistent in terms of reducing realised risk ( $80.5 \%$ of the time overall). However, combining all methods, RMT filtering caused increased realised risk by $20 \%$ or more around $5.5 \%$ of the days, with correlation and covariance filtering accounting for roughly half of this each. KR filtering increased realised risk by $20 \%$ or more the least often ( $2.0 \%$ of the days). On $0.3 \%$ of the days, RMT filtering increased realised risk by $60 \%$ or more, at least doubling it on two of those days. This shows that, while RMT filtering reduced realised risk on average, and on the majority of days, all the tested filters had the capacity to increase realised risk, and some marked increases were observed.

Figure 6 shows the values of the decay factors chosen through time by the for- 
ward validation. The decay factor chosen for the unfiltered series, not shown, was always the maximum tested (0.999). All the decay factors chosen using forward validation were higher than the 0.97 suggested by Riskmetrics [1], especially those for filtering covariance.
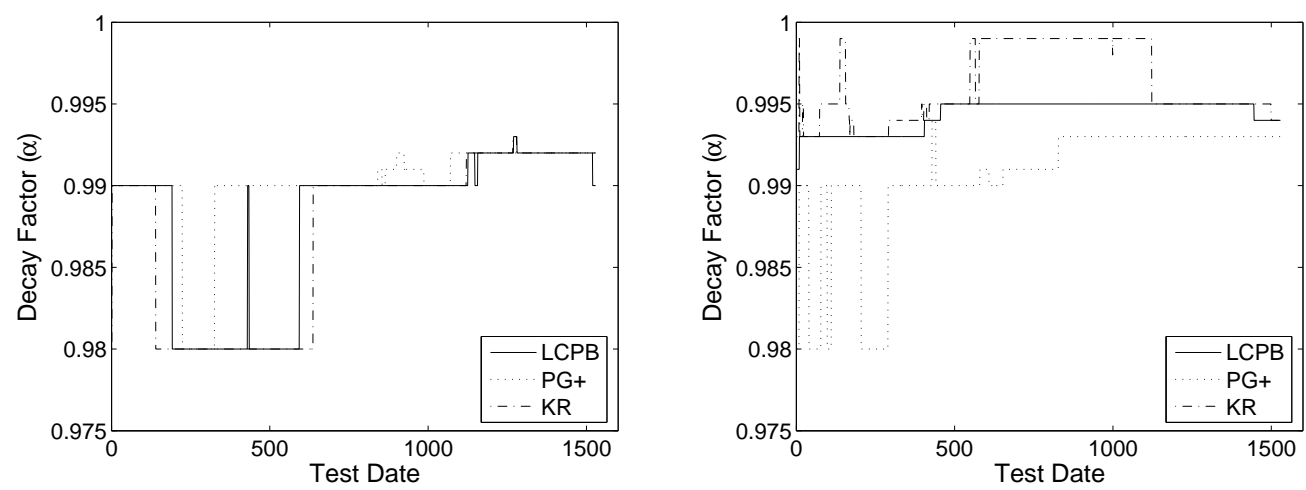

Fig. 6. Optimal forward validation decay factor values for filtering the correlation matrix (left) and the covariance matrix (right). The range of decay factors seen here is higher in all cases than the Riskmetrics [1] value of 0.97 .

\section{Conclusions}

In this work we have studied application of RMT filters to the optimisation of financial portfolios. Broadly, our results for our novel filter are in agreement with previous results [16], that RMT-based filtering can improve the realised risk of minimum risk portfolios. Based on Krzanowski stability, the filter extends that which we developed earlier, Sharifi et al. [17], and offers improvements in terms of risk and stability compared to other RMT filters tested.

Using forward validation, RMT filters were found to reduce mean realised risk, overall, in all cases tested. However, in some individual years this was not the case. When considering individual days, RMT filtering was found to reduce realised risk for $72.3 \%$ of the test cases $(74.3 \%$ for filtering correlation and $80.5 \%$ for the best filter). However, it was also found capable of increasing realised risk for all types of filters, substantially in some cases. The overall best method, out-of-sample, was exponentially weighted covariance, with our Krzanowski stability-based filter applied to the correlation matrix. This method also showed good consistency for reducing risk on an annual, monthly and daily basis.

When examined in-sample, filtering covariance, rather than correlation, produced lower risk portfolios in some cases, but on average filtering correlation 
generated lower realised risk out-of-sample. In-sample tests also supplied some evidence, in the form of local optima, to support the Riskmetrics [1] recommended decay factor of 0.97 . However, the optimal out-of-sample decay factors, for both filtered and unfiltered forecasts, were higher in all cases than those suggested by Riskmetrics [1], with those for the latter approaching a value of $\alpha=1$.

While this work focuses on the realised risk (of the forecast minimum risk portfolio) as the measure for assessing optimal performance, we note that a different choice of metric can affect the results. For example, minimising portfolio risk and obtaining the best forecast of portfolio risk do not necessarily result in the same choice of models or parameters. This limits wide ranging conclusions on best choice of filter or parameter values. Instead, these results suggest that RMT filtering has the potential to offer risk reduction for portfolio optimisation applications.

\section{Acknowledgements}

We would like to thank the Irish Research Council for Science, Engineering and Technology for supporting this research under the Embark Scholarship Scheme.

\section{A LCPB and PG + Filtering Methods}

\section{A.1 LCPB Filtering Method}

The method described by Laloux et al. [15] (and referred to here as LCPB) takes the set, $\Lambda$, of eigenvalues of some $N \times N$ matrix, $\mathbf{M}$, and the corresponding eigenvectors, $\mathbf{E}$, and defines the subset

$$
\Lambda_{\text {noisy }}=\left\{\lambda \in \Lambda: \lambda \leq \lambda_{+}\right\}
$$

of noisy eigenvalues, where $\lambda_{+}$is some maximum eigenvalue predicted by RMT. A set of filtered eigenvalues are then defined as

$$
\Lambda_{\text {filtered }}=\Lambda_{\text {new }} \cup\left(\Lambda-\Lambda_{\text {noisy }}\right)
$$


where

$$
\Lambda-\Lambda_{\text {noisy }}=\left\{\lambda \in \Lambda: \lambda \notin \Lambda_{\text {noisy }}\right\}
$$

are the eigenvalues assumed to contain information and

$$
\Lambda_{\text {new }}=\left\{\lambda_{i}: \lambda_{i}=\bar{\Lambda}_{\text {noisy }} \forall i=1, \ldots, n\right\}
$$

where $n$ is the number of elements in $\Lambda_{\text {noisy }}$ and $\bar{\Lambda}_{\text {noisy }}$ is the mean of all the elements of $\Lambda_{\text {noisy }}$. In other words, the noisy eigenvalues are all replaced with their mean. These filtered eigenvalues $\Lambda_{\text {filtered }}$ are then combined, via the eigen decomposition theorem ${ }^{6}$, with the original eigenvectors, $\mathbf{E}$, to construct a filtered matrix

$$
\mathbf{M}_{\text {filtered }}=\mathbf{E D}_{\text {filtered }} \mathbf{E}^{-1}
$$

where $\mathbf{D}_{\text {filtered }}$ is a matrix with $\Lambda_{\text {filtered }}$ on the main diagonal and zeroes everywhere else. Replacing the noisy eigenvalues by the mean noisy eigenvalue means that the trace of $\mathbf{M}_{\text {filtered }}$ is equal to the trace of $\mathbf{M}$.

\section{A.2 $P G+$ Filtering Method}

As described by Plerou et al. [9] (and referred to here as PG+), this method is the same as the LCPB method, except that the noisy eigenvalues are all replaced by zeroes. Then, after the filtered matrix $\mathbf{M}_{\text {filtered }}$ is built, its main diagonal is set to be equal to that of the original matrix $\mathbf{M}$, thus preserving the trace.

$\overline{{ }^{6}}$ Let $\mathbf{M}$ be a square matrix and let $\mathbf{E}$ be the matrix of its eigenvectors. If $\mathbf{E}$ is a square matrix then $\mathbf{M}=\mathbf{E} \mathbf{D E}^{-1}$ where $\mathbf{D}$ is a diagonal matrix containing the corresponding eigenvalues on the main diagonal. [21] 


\section{References}

[1] J. P. Morgan and Reuters. Riskmetrics technical document. Technical report, http://www.riskmetrics.com/techdoc.html, 1996.

[2] H. Markowitz. Portfolio Selection: Efficient Diversification of Investments. Wiley, New York, 1959.

[3] J.P. Bouchaud and M. Potters. Theory of Financial Risks - From Statistical Physics to Risk Management. Cambridge University Press, U.K., 2000.

[4] M. Dacorogna, R. Gençay, U. Muller, R. Olsen, and O. Pictet. An Introduction to High-Frequency Finance. Academic Press, U.S., 2001.

[5] F. Dyson. Statistical theory of the energy levels of complex systems i, ii and iii. J. Math. Phys., 3(1):140-175, 1962.

[6] F. Dyson and M. Mehta. Statistical theory of the energy levels of complex systems iv. J. Math. Phys., 4(5):701-712, 1963.

[7] M. Mehta and F. Dyson. Statistical theory of the energy levels of complex systems v. J. Math. Phys., 4(5):713-719, 1963.

[8] M. Mehta. Random Matrices. Academic Press, New York, 1991.

[9] V. Plerou, P. Gopikrishnan, B. Rosenow, L.A.N. Amaral, T. Guhr, and H.E. Stanley. Random matrix approach to cross correlations in financial data. Phys. Rev. E, 65(6):066126, 2002.

[10] V. Plerou, P. Gopikrishnan, B. Rosenow, L.A.N. Amaral, and H.E. Stanley. Universal and nonuniversal properties of cross correlations in financial time series. Phys. Rev. Lett., 83(7):1471-1474, 1999.

[11] V. Plerou, P. Gopikrishnan, B. Rosenow, L.A.N. Amaral, and H.E. Stanley. Econophysics: financial time series from a statistical physics point of view. Physica A, 279:443-456, 2000.

[12] V. Plerou, P. Gopikrishnan, B. Rosenow, L.A.N. Amaral, and H.E. Stanley. A random matrix theory approach to financial cross-correlations. Physica A, 287:374-382, 2000 .

[13] V. Plerou, P. Gopikrishnan, B. Rosenow, L.A.N. Amaral, and H.E. Stanley. Collective behavior of stock price movements - a random matrix theory approach. Physica A, 299:175-180, 2001.

[14] L. Laloux, P. Cizeau, J.P. Bouchaud, and M. Potters. Noise dressing of financial correlation matrices. Phys. Rev. Lett., 83(7):1467-1470, 1999.

[15] L. Laloux, P. Cizeau, M. Potters, and J.P. Bouchaud. Random matrix theory and financial correlations. Int. J. Theor. Appl. Finance, 3(3):391-397, 2000. 
[16] S. Pafka, M. Potters, and I. Kondor. Exponential weighting and random-matrixtheory-based filtering of financial covariance matrices for portfolio optimization. To appear in Quant. Fin.; e-print cond-mat/0402573, 2004.

[17] S. Sharifi, M. Crane, A. Shamaie, and H. J. Ruskin. Random matrix theory for portfolio optimization: a stability approach. Physica A, 335:629-643, 2004.

[18] W.J. Krzanowski. Sensitivity of principal components. J. Royal Stats. Soc. B, 46(3):558-563, 1984.

[19] A.M. Sengupta and P. P. Mitra. Distributions of singular values for some random matrices. Phys. Rev. E, 60(3):3389-3392, 1999.

[20] M. Potters, J.P. Bouchaud, and L. Laloux. Financial applications of random matrix theory: Old laces and new pieces. In Proceedings of the Kraków conference on Applications of Random Matrix Theory to Economy and Other Complex Systems, arXiv:physics/0507111, 2005.

[21] G. Strang. Linear Algebra and its Applications. Academic Press, New York, 1980 . 